\title{
2loolf 27ajt
}

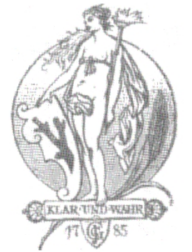

B. J. Böjchen'ithe Derlagshandlung $\$$ 



\section{SSotthold Ephraim Reffing's}

\section{$\mathfrak{f a m m} \mathfrak{m} \mathfrak{i} \mathfrak{d} \mathfrak{e} \mathfrak{S}_{\mathfrak{d}} \mathfrak{r} \mathfrak{i} \mathfrak{f} \mathfrak{t} \mathfrak{n}_{\text {. }}$}

$\mathfrak{Z}$ htgehnter $\mathfrak{B a n d}$.

\section{$\mathfrak{B} \mathfrak{e} \mathfrak{\mathfrak { l }} \mathfrak{i} \mathfrak{n}_{*}$}

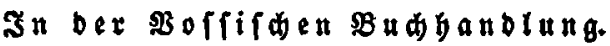

$182 \%$ 
